\title{
Utilization of fuzzy controller for laboratory scale convective fruit dryers
}

\begin{abstract}
In the present study, a fruit dryer system that is controlled based on fuzzy logic is presented. A laboratory scale cabinet was developed which includes four sensors in different lengths for monitoring the cabin temperature and humidity. Fuzzy base controller is a new monitoring technique in food industrial machines that utilize sensors captured values as its input parameters to make a suitable decision according to temperature values. Furthermore, to implement the fuzzy system, a microcontroller base monitoring system is developed. Microcontroller captured temperature samples and converted them in to digital values. Output of the fuzzy controller will control the speed of the fan and power of the heater. Several performed results indicated the amenability of the proposed monitoring system as a drying machine main controller in different drying curves. Fluctuation of the cabin temperature with fuzzy control was smoother than non-fuzzy control. Nevertheless, fuzzy control has a significant influence on the power consumption as well.
\end{abstract}

Keyword: Convective dryer; Fruit dryer; Fuzzy control; Heat transfer; Microcontroller 